\title{
Modelo de referencia operacional aplicado a una empresa de servicios de mantenimiento*
}

\author{
Herrera Vidal, German** \\ Herrera Vega, Juan Carlos ${ }^{* * *}$
}

\section{Resumen}

El éxito en las empresas depende de que sus procesos se encuentren alineados e integrados, permitiendo gestionar efectivamente la cadena de suministros. El objetivo del presente artículo es analizar un modelo de referencia operacional, encaminado a determinar oportunidades de mejora e identificar mejores prácticas. Por esto, la investigación es de tipo descriptiva, basada en la metodología planteada por el modelo, mediante el análisis de cinco procesos básicos como planeación, aprovisionamiento, producción, distribución y devoluciones. Se revisó o la literatura, siendo el principal modelo de referencia encontrado el desarrollado por el consejo de cadena de suministros. Se desarrolló la caracterización y análisis de los procesos logísticos y por último la evaluación de las fallas en la ejecución de las actividades. Los hallazgos encontrados evidencian poder mejorar los rendimientos actuales de los indicadores de gestión. A partir de esto se concluye que adoptar esta metodología permite administrar eficientemente la cadena de suministro de la empresa del sector, logrando beneficiar la rentabilidad de los inversionistas y la satisfacción de los clientes.

Palabras clave: Modelo de referencia operacional logística; cadena de suministro; planeación; aprovisionamiento; producción; distribución; devoluciones.

Recibido: 30-08-15. Aceptado: 05-06-16

* En esta investigación, se extiende especial agradecimiento a las ingenieras Nuris Vanessa De Horta y Blanca Mercedes Piña por su gestión para la recopilación de la información primaria y el apoyo dispensado en las visitas técnicas en la empresa de servicios de mantenimiento eléctrico industrial de la ciudad de Cartagena. De igual forma al Dr. Ricardo Vivas Reyes por sus consejos y orientaciones en la estructura del trabajo. Por último a la Fundación Universitaria Tecnológico Comfenalco por el apoyo de su grupo científico.

** Magíster en Ingeniería con énfasis en Industrial (UTB, Colombia). Docente Investigador del Grupo de Investigación CIPTEC. Fundación Universitaria Tecnológico Comfenalco. Colombia. Email: ghermany@hotmail.com

*** Magíster en Ingeniería Industrial (USB, Colombia). Docente Investigador del Grupo de Investigación CIPTEC. Fundación Universitaria Tecnológico Comfenalco. Cartagena. Colombia. Email: juancarlos.herreravega@gmail.com 


\title{
Methodology Based on SCOR Model Applied to a Maintenance Services Company
}

\begin{abstract}
The success of the companies depends that their processes have to be aligned and integrated, allowing the possibility to effectively manage the supply chain. The objective of this article is to apply an operational reference model aimed at identifying opportunities for improvement and identify best practices. For this reason, research is descriptive type based on the methodology proposed by the model, by analyzing five basic processes such as planning, procurement, production, distribution and returns. This involved a literature review, in which the main reference model found is the one developed by the Council of Supply Chain (SCC). Then, it was developed the characterization and analysis of logistics processes and finally assessing failures in the implementation of activities. The findings show to improve current management indicators. From this, it is concluded that adopting this methodology allows efficiently managing the supply chain of the company in the sector, achieving profitability benefit for investors and customer satisfaction.
\end{abstract}

Keywords: Supply chain; operations reference model (SCOR); logistics.

\section{Introducción}

La competitividad que existe en la actualidad debido a los efectos de la globalización, ha generado cambios acelerados en la economía. Algunas empresas tienen la capacidad de poder mantener el ritmo, adaptarse, prosperar y mantenerse en el creciente mundo incierto. Todo esto gracias a que tienen la capacidad de analizar, anticiparse y comprender la tendencia y todos los futuros cambios que se avecinan en el mercado. Ahora bien, para ofrecer nuevos bienes y servicios, y adaptar sus negocios rápidamente a las exigencias del contexto, satisfaciendo las necesidades de sus clientes, es fundamental considerar elementos clave como la fiabilidad en el cumplimiento, flexibilidad y velocidad de atención.

Para esto todos los actores de la cadena deben integrar sus procesos desde los clientes aguas arriba hasta llegar a los proveedores, y es aquí donde toma fuerza el termino de gestión de cadena de suministros, el cual se ha convertido en un concepto fundamental para que las empresas mejoren las relaciones con clientes y proveedores, desarrollando ventajas competitivas.

Hoy en día, los modelos de negocios del mundo moderno están en continuo desarrollo, entrando en nuevas tendencias y economías, nuevas áreas industriales y hasta nuevos modelos en un entorno de alta competitividad (Herrera, 2014:2). Es por esto que existen diferentes tipos de modelos de gestión para las cadenas de suministros, dentro de los que se encuentran modelos conceptuales y analíticos. Los conceptuales, consisten en herramientas descriptivas, que subrayan los aspectos principales y las variables relevantes implicadas en un problema específico o estructuras empíricas que proponen directrices para abarcar los problemas de la cadena de suministros. La gran mayoría están pensados 
para resolver problemas de configuración y de coordinación, cuyo objetivo final es el logro de un enfoque orientado a los procesos en la cadena de suministros, entre ellos se encuentran la matriz de planificación de la cadena de suministro (SCP), el modelo de planificación colaborativa, previsión y reabastecimiento (CPFR), el modelo de referencia de operaciones de la cadena de suministro (SCOR).

Este último, también llamado SCOR, es una herramienta de gestión para representar, analizar y diagnosticar cadenas de suministro. Para el Supply Chain Council (2006), la metodología SCOR ayuda a las compañías a detectar los problemas de su cadena de suministro, identificando según sus objetivos y mejoras en su desempeño.

Por otro lado, los modelos analíticos se basan en diferentes técnicas de la investigación operativa, entre ellas, la programación matemática, teoría de inventarios, teoría de la decisión, procesos de Markov y procesos jerárquicos, entre otros (Lario y Pérez, 2005:2).

En este sentido, se pretende responder a una necesidad por parte de una empresa que presta servicios de mantenimiento eléctrico, encaminada a determinar oportunidades de mejora e identificar mejores prácticas en su cadena de suministros. Donde a partir de la gerencia se han ejecutado planes para el buen manejo de la cadena productiva, estos tienen como prioridad los procesos de manufactura, dejando de lado los otros procesos vitales dentro de la cadena, como la gestión de los pedidos, la gestión de servicio hacia los clientes, la gestión de la demanda, la gestión de compras, la gestión de los costos y manejo de los inventa- rios, la gestión de la distribución, entre otros. Lo que ha contribuido a que no toda la cadena funcione armónicamente y que los esfuerzos emprendidos no generen los impactos deseados.

Dado lo anterior, se tomó como referente el modelo SCOR, por lo que su metodología permite identificar características habilitadoras para poder solucionar la problemática presentada en la presente investigación.

Desde el marco de aplicabilidad del modelo de referencia operacional (SCOR) primeramente se hace una caracterización de la cadena identificando los agentes, las relaciones existentes desde los proveedores de segundo nivel hasta los clientes y el estado actual, luego se hace una evaluación y un análisis a partir de una serie de indicadores en cada uno de los niveles permitiendo gestionar la cadena en cada uno de sus procesos.

Desde el punto de vista metodológico, se realizan entrevistas estructuradas al gerente, supervisor y jefe de producción, adicional a esto se estructuraron e intervinieron encuestas dirigidas a los operarios partícipes en el proceso. Por tratarse de una pequeña empresa que tiene una población menor a cincuenta (50) trabajadores, no se requirió definir el tipo de muestreo, por lo que la muestra es igual a la población.

\section{Gestión de la cadena de suministros}

El concepto de cadena de suministro aparece claramente en los trabajos de Forrester (1961), cuando sugirió que el éxito de las empresas dependía de la interacción entre los flujos de información, 
Modelo de referencia operacional aplicado a una empresa de servicios... Herrera Vidal, German y Herrera Vega, Juan Carlos

materiales, pedidos, dinero, mano de obra y equipos. La comprensión y control de estos flujos es el trabajo principal en la gestión. En sus experimentos de simulación, a través de un modelo de cadena de suministro compuesto por cuatro niveles (fábrica, almacén, distribuidor y minorista), estudió el efecto bullwhip como uno de los efectos indeseados que el encadenamiento multi-etapas puede producir.

Después del trabajo de Forrester fueron apareciendo otras definiciones de cadena de suministro. Burns y Sivazlian (1978:182), definieron a la cadena de suministro como el conjunto de empresas que actúan en el diseño, ingeniería, mercadeo, fabricación y distribución de bienes y servicios a los consumidores finales. Basado en las diferentes actividades que se desarrollan en una cadena de suministro, Stevens (1989:3) y Lee y Billington (1993:836), expresaron que: "una cadena de suministro está conectada por una serie de actividades, como la planificación, coordinación, control de materiales y productos terminados, de acuerdo a las necesidades de los clientes", lo cual tiene que ver con dos flujos distintos dentro de la organización, uno de material y otro de información.

En los inicios del siglo XX, Min y Mentzer (2000:766), definen la cadena de suministro como "una integración funcional entre empresas que implementan acciones para gestionar el flujo dentro del canal de distribución desde el proveedor hasta el cliente final, en la cual todos deben contribuir en el aumento de competitividad". Chandra y Kumar (2000:101), la consideraron como un sistema de relaciones entre varias empresas que permite consensuar la necesidad de cubrir la demanda del cliente final y buscar el crecimiento del beneficio.

El término Supply Chain Management (SCM) permitió expresar la necesidad de integrar los procesos clave del negocio, desde los proveedores originales que proveen productos, servicios e información a través de la agregación de valor para el consumidor y los otros clientes (Lambert et al. 1998:2). En donde se representa una cadena de suministro simplificada, mostrando los flujos y los ocho procesos clave de gestión de la cadena de suministro como: (i) Gestión de las relaciones con los clientes, (ii) Gestión de los servicios a los clientes, (iii) Gestión de la demanda, (iv) Gestión de los pedidos, (v) Gestión del flujo la manufactura, (vi) Gestión de las relaciones con los proveedores, (vii) Desarrollo de productos y comercialización y (viii) Gestión de los retornos.

En términos sencillos, la cadena de suministro puede ser entendida como un conjunto de empresas compuestas por proveedores, fabricantes, distribuidores y clientes, conectadas entre sí por una serie flujos, procesos y enfoques que permiten tener una visión completa de lo que ocurre en la cadena.

\section{Modelo de Referencia de Operaciones (SCOR)}

Hoy en día las empresas deben contar con alguna metodología que sea capaz de evaluar el estado de sus cadenas y brindar herramientas para el correcto diagnóstico de sus operaciones. Para ello, el principal modelo de referencia operacional encontrado en la literatura es el modelo planteado por el Supply Chain Council (SCC). 
Fue desarrollado en el año 1996 por el Consejo de la Cadena de Suministro, también llamado SCC (Supply Chain Council) una corporación independiente sin fines de lucro, como una herramienta de diagnóstico estándar para la gestión de la cadena de suministro en cualquier organización (Bolstorff y Rosenbaum, 2012:9).

El Supply Chain Council (2006:2), define al SCOR como "un modelo desarrollado para describir todas las actividades que una empresa ejecuta mediante fases para satisfacer la demanda de los clientes. El modelo se organiza teniendo en cuenta los cinco procesos básicos que se llevan a cabo en la empresa: planeación, aprovisionamiento, producción, distribución y devoluciones". En su análisis Calderón y Lario (2005:2) concluyen que el modelo es una herramienta de representación, análisis y configuración de cadenas de suministro que proporciona un marco único que relaciona los procesos de la empresa, los indicadores de gestión, las mejores prácticas y la reingeniería en una red unificada para facilitar la comunicación entre las diferentes organizaciones y procesos que conforman la cadena.

El Diagrama 1 muestra cómo el alcance del modelo SCOR abarca todos los procesos desde los proveedores hasta los clientes; por lo tanto se constituye en un medio para lograr un análisis profundo y también el mejoramiento total de la cadena de suministro.

El modelo contiene diversas secciones organizadas en torno a los cinco procesos primarios que son: Planificación (Plan), Abastecimiento (Source), Producción (Make), Distribución (Deliver) y Devoluciones (Return) (Supply Chain Council, 2010).

Al analizar los procesos de la cadena de suministro utilizando los bloques anteriores, el modelo puede ser utilizado para describir cualquier cadena, desde

\section{Diagrama 1 \\ Modelo referencia de operaciones en la cadena de suministros}

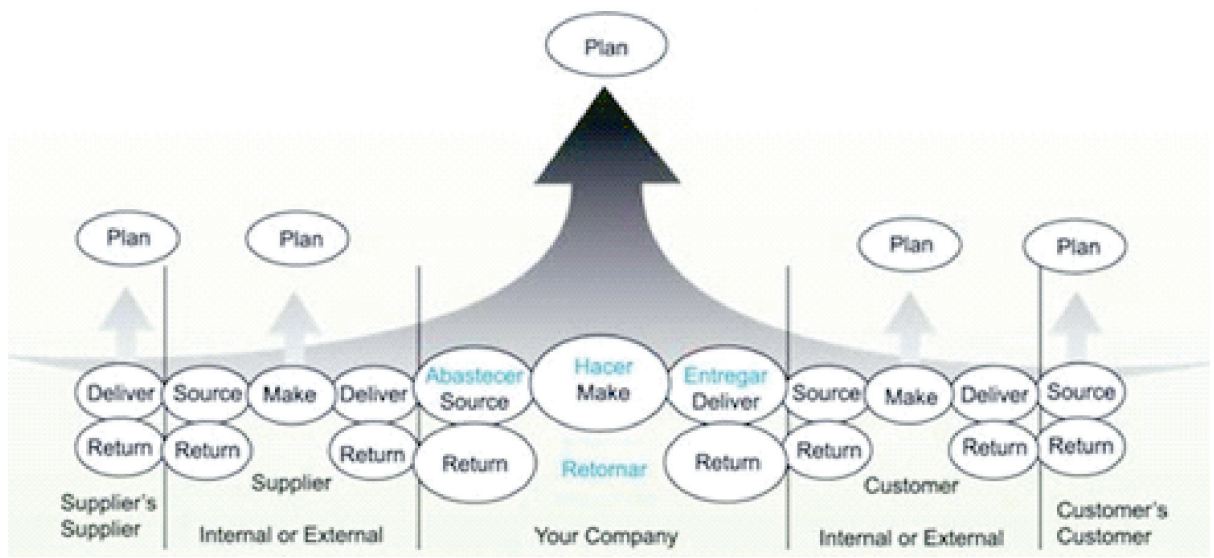

Fuente: Calderón y Lario (2005), 
Modelo de referencia operacional aplicado a una empresa de servicios... Herrera Vidal, German y Herrera Vega, Juan Carlos

las más simples hasta las más complejas, utilizando una serie de definiciones comunes.

A su vez el modelo está enfocado en tres niveles de proceso: Nivel 1, el cual apunta a la gerencia y sirve para tomar decisiones sobre la estrategia de la cadena; Nivel 2, que apunta a la forma de gestionar y estructurar la cadena; Nivel 3, que pone foco en cada uno de los procesos desarrollados en la cadena (Supply Chain Council, 2010). Adicionalmente, existe un Nivel 4, que corresponde al nivel en el cual la empresa interioriza las lecciones aprendidas del resto de los niveles y comienza a aplicar prácticas y métricas específicamente diseñadas para su empresa. Este nivel está excluido del alcance del modelo SCOR por ser justamente una tarea individual de cada empresa (Huan et al. 2004:24).
En los tres primeros niveles, el modelo SCOR aporta indicadores clave de rendimiento (KPI's). Estos Indicadores se dividen en: (i) Fiabilidad en el Cumplimiento, (ii) Flexibilidad, (iii) Velocidad de Atención, (iv) Costo y (v) Activos (Calderón y Lario, 2005:2) (Cuadro 1).

\subsection{Nivel superior - Tipos de Procesos}

En este nivel se define el alcance y contenido del modelo SCOR, se analizan las bases de competición y se establecen los objetivos de rendimiento competitivo de los procesos de aprovisionamiento, producción y distribución (Supply Chain Council, 2004).

Estas métricas ayudan a dar una visión estratégica de los diferentes atributos de desempeño, dado que no pueden

\section{Cuadro 1}

Descripción de los indicadores de gestión del nivel I

\begin{tabular}{|c|c|c|}
\hline Indicador & Definición & Medición \\
\hline Fiabilidad & $\begin{array}{l}\text { Mide el desempeño de la cadena de suministros } \\
\text { en el proceso de entrega de acuerdo a las condi- } \\
\text { ciones aptas, con la calidad requerida, con la do- } \\
\text { cumentación e información pertinente. }\end{array}$ & $\begin{array}{l}\text { Cumplimiento de entrega de } \\
\text { servicios }\end{array}$ \\
\hline $\begin{array}{l}\text { Velocidad de } \\
\text { Atención }\end{array}$ & $\begin{array}{l}\text { Se refiere a la velocidad con la cual la cadena de } \\
\text { suministros provee bienes o servicios a los clien- } \\
\text { tes tanto internos como externos. }\end{array}$ & $\begin{array}{l}\text { Ciclo de tiempo de } \\
\text { cumplimiento de una entrega } \\
\text { de servicios }\end{array}$ \\
\hline Flexibilidad & $\begin{array}{l}\text { Hace referencia a la agilidad o flexibilidad de la ca- } \\
\text { dena de suministros ante cambio en el mercado, } \\
\text { con el propósito de mantener o ganar ventajas } \\
\text { competitivas. }\end{array}$ & $\begin{array}{l}\text { Flexibilidad en porcentaje de la } \\
\text { cadena de suministros hacia } \\
\text { arriba }\end{array}$ \\
\hline Costos & $\begin{array}{l}\text { Hace referencia a los costos asociados a la opera- } \\
\text { ción y gestión de la cadena de suministros. }\end{array}$ & $\begin{array}{l}\text { Costo total de gestión de la } \\
\text { cadena de suministros }\end{array}$ \\
\hline $\begin{array}{l}\text { Gestión de } \\
\text { activos }\end{array}$ & $\begin{array}{l}\text { Miden la efectividad financiera de la cadena de su- } \\
\text { ministros, el manejo que se le da a los activos fijos } \\
\text { o al capital de trabajo, entre otras. }\end{array}$ & $\begin{array}{l}\text { Retorno de los activos fijos de } \\
\text { la cadena de suministros }\end{array}$ \\
\hline
\end{tabular}

Fuente: Elaboración propia. 
ser medidos, se evalúan teniendo en cuenta el comportamiento y el rendimiento dentro de la organización.

Los indicadores de nivel 1 son medidas de alto nivel que recorren múltiples procesos SCOR. Los indicadores de nivel 1 no se relacionan necesariamente con todos los procesos de nivel 1 (plan, aprovisionamiento, manufactura, distribución, devolución). Los tres primeros (fiabilidad en el cumplimiento, flexibilidad y velocidad de atención) son puntos de vista externos, mientras que costo y activo son puntos de vista internos (Calderón y Lario, 2005:3).

Posteriormente, los valores de los indicadores del nivel superior se analizan en una tabla de valores objetivos de la cadena de suministros, de manera que se puedan identificar posibles falencias o problemas dentro de la cadena, permitiendo poder realizar un plan de mejoramiento a nivel global

\subsection{Nivel de Configuración - Categorías de Procesos}

Según Patiño (2008), en el nivel de configuración o segundo nivel, cada proceso puede ser descrito teniendo en cuenta tres tipos: (i) Planificación, en el cual se ajustan los recursos esperados para satisfacer los requerimientos de la demanda esperada, e involucran diferentes características como el balance de la demanda agregada y la cadena, la consistencia en el horizonte de planeación y el tiempo de respuesta de la cadena de suministros. (ii) Ejecución, es un proceso desencadenado por la planificación o por la actual demanda que cambia el estado de material de bienes, generalmente im- plica secuenciación, transformación de producto y movimiento de producto al siguiente proceso. (iii) Apoyo, es un proceso que prepara, mantiene o maneja información de los que dependen los procesos de planificación y ejecución.

En el segundo nivel se consideran 26 categorías procesos, que son las categorías principales que permiten configurar la cadena de prácticamente cualquier empresa, corresponden 5 a Plan, 3 a Aprovisionamiento, 3 a Manufactura, 4 a Distribución, 6 a Devolución (3 de Aprovisionamiento y 3 de Distribución), y 5 a Apoyo. Las cinco primeras son de tipo planificación, las 16 intermedias son de tipo ejecución y las 5 últimas son de tipo apoyo (Calderón y Lario, 2005:4).

Las tres categorías en las que se subdividen aprovisionamiento, manufactura y distribución son fabricación contra almacén (Make-to-Stock), fabricación bajo pedido (Make-to-Order) y diseño bajo pedido (Enginer-to-Order) pero la distribución o entrega tiene una cuarta categoría que es producto de venta al por menor (Retail Produce). Devolución (Return) a su vez tiene tres categorías: producto defectuoso, producto para mantenimiento general y reparación, y producto en exceso (Patiño, 2008).

En este nivel, la cadena de suministro debe representarse usando las 26 categorías de procesos conforme a su estado actual (AS-IS), tanto geográficamente, como también con diagramas de hilos, para después establecer las especificaciones de diseño de su nueva cadena de suministros y poder reconfigurarla al estado deseado (TO BE) empleando nuevamente los dos tipos de gráficos mencionados (Calderón y Lario, 2005:5). 
Modelo de referencia operacional aplicado a una empresa de servicios... Herrera Vidal, German y Herrera Vega, Juan Carlos

Los indicadores de nivel 2, de igual forma contemplan los atributos del modelo SCOR, como son: (i) Fiabilidad en el Cumplimiento, (ii) Capacidad de Respuesta, (iii) Agilidad, (iv) Costos y (v) Manejo de activos. Permitiendo realizar un diagnóstico a las métricas del Nivel 1 , ayudando a identificar errores o vacíos que se presenten en el nivel anterior (Cuadro 2).

\subsection{Nivel de Elementos de Procesos - Descomposición de los Procesos}

En el tercer nivel se representan los distintos procesos de la cadena de suministros de manera más detallada descomponiendo las categorías en elementos de procesos (Process elements). Estos se presentan en secuencia lógica (con rectángulos y flechas) con entradas (inputs) y salidas (outputs) de información y materiales. Además, en el nivel 3 se evalúa el rendimiento de cada proceso y elemento mediante índices (Process performance metrics), de manera que se encuentren las diferencias de rendimiento entre los procesos y elementos de la cadena de suministros (Calderón y Lario, 2005:5).

\subsection{Casos de aplicación empresarial mediante el modelo SCOR}

La aplicabilidad del modelo SCOR, ha sido objeto de múltiples trabajos, buscando siempre la solución de diversos problemas y la excelencia operacional de la cadena de suministros (Cuadro 3 ). De los artículos revisados se analizan los diferentes sectores en los cuales se aplicó el modelo SCOR (Cuadro 4).
Dado lo anterior se plantea un caso de estudio interesante producto de la poca aplicabilidad, como es el de una empresa del sector eléctrico industrial, donde se presentan problemas relacionados con el cumplimiento de las ordenes y la calidad del producto final.

\section{Generalidades del caso de estudio}

Para la aplicación del modelo SCOR se tomó como referente una empresa dedicada exclusivamente a la prestación de servicios de reparación y mantenimiento de equipos eléctricos en la ciudad de Cartagena,

Dentro de estos servicios se encuentran: (i) Rebobinado de motores eléctricos, siendo este el servicio por el cual es reconocida la compañía, este representa en promedio un $70 \%$ de la totalidad de los servicios prestados en un periodo de tiempo determinado. (ii) Mantenimiento de motores eléctricos, este servicio comprende desde el lavado del devanado del estator hasta el cambio de rodamientos del eje del motor, pasando por la reparación y restauración de piezas y demás partes del equipo, corresponde a un $15 \%$ de la totalidad de los servicios prestados. (iii) Servicios de mecanizados, son solicitados de manera independientes por los clientes, es decir, envían solo la parte del eje de motor y/o equipo eléctrico para reparación. (iv) Servicios en campo, consisten en la realización de actividades de desmontaje, desconexión, montaje, conexión, limpieza, cambio de rodamientos y realización de pruebas eléctricas a equipos en campo, en las instalaciones de la empresa cliente, servicio 


\section{Cuadro 2}

Descripción de los indicadores de gestión del nivel II

\begin{tabular}{|c|c|c|}
\hline Indicador & Descripción & Cálculos Métricos \\
\hline \multirow{3}{*}{ Fiabilidad } & $\begin{array}{l}\text { Porcentaje de servicios } \\
\text { entregados en su totalidad }\end{array}$ & $\begin{array}{l}\text { (Número de equipos entregados en su totalidad/ equi- } \\
\text { pos entregados) } \times 100\end{array}$ \\
\hline & $\begin{array}{l}\text { Rendimiento de entrega al } \\
\text { cliente en la fecha pactada }\end{array}$ & $\begin{array}{l}\text { (Número de servicios o equipos entregados a tiempo } \\
\text { /número de equipos entregados) } \times 100\end{array}$ \\
\hline & Perfecto estado & $\begin{array}{l}\text { (Número de servicios o equipos entregados en perfec- } \\
\text { to estado/número de equipos entregados) }\end{array}$ \\
\hline \multirow[b]{2}{*}{$\begin{array}{l}\text { Velocidad } \\
\text { de } \\
\text { Atención }\end{array}$} & $\begin{array}{l}\text { Ciclo de tiempo del } \\
\text { suministro }\end{array}$ & $\begin{array}{l}\text { (Identificación de las fuentes de suministro del proveedor } \\
\text { (tiempo de cotización + realización del pedido + envío del } \\
\text { insumo +recibo del insumo + pago de proveedores) }\end{array}$ \\
\hline & $\begin{array}{l}\text { Ciclo de tiempo } \\
\text { de entrega }\end{array}$ & $\begin{array}{l}\text { Tiempo estimado de los procesos para la entrega (ge- } \\
\text { neración de la orden interna de trabajo + determinar y } \\
\text { asignar los recursos para la fecha de entrega + tiempo } \\
\text { de liberación del equipo o servicio + coordinación de la } \\
\text { entrega) }\end{array}$ \\
\hline \multirow[t]{4}{*}{ Flexibilidad } & $\begin{array}{l}\text { Flexibilidad en días de las } \\
\text { compra hacia arriba }\end{array}$ & $\begin{array}{l}\text { Número de días necesarios para lograr un aumento im- } \\
\text { previsto sostenible en la cantidad de insumos }\end{array}$ \\
\hline & Costo de planificar & $\begin{array}{l}\text { Los gastos de recursos (sueldos de jefe de logística y al- } \\
\text { macenistas) y de funcionamiento del proceso basados } \\
\text { en relaciones comerciales con los proveedores suma de } \\
\text { costos de la planificación y los de administración }\end{array}$ \\
\hline & & $\begin{array}{l}\text { Gestión de proveedores de material = planificación + } \\
\text { negociación de proveedores y evaluación + etc. }\end{array}$ \\
\hline & Costo de comprar & $\begin{array}{l}\text { Adquisición de material y gestión de ofertas y cotizacio- } \\
\text { nes }=\text { recibir }+ \text { pedidos de control del material entrante } \\
+ \text { almacenamiento de los materiales }\end{array}$ \\
\hline \multirow{4}{*}{ Costos } & & $\begin{array}{l}\text { Autorización de pago de abastecimiento + gestión de } \\
\text { pago según forma (crédito o contado) }\end{array}$ \\
\hline & & $\begin{array}{l}\text { La gestión de ventas = recepción del servicio + trans- } \\
\text { porte del equipo + facturación }\end{array}$ \\
\hline & Cos & $\begin{array}{l}\text { La gestión de clientes = gestión post venta (asesorías + } \\
\text { garantía) }\end{array}$ \\
\hline & Costo de devolver & $\begin{array}{l}\text { Costo para devolver un equipo = costo de la recepción } \\
\text { del servicio + costo de la revisión + transporte del equi- } \\
\text { po (si aplica) }\end{array}$ \\
\hline \multirow{2}{*}{$\begin{array}{l}\text { Gestión de } \\
\text { activos }\end{array}$} & Cuentas por pagar & $\begin{array}{l}\text { Cuentas pendientes de pago contenidas en el balance } \\
\text { general }\end{array}$ \\
\hline & Inventario & Inventarios disponibles contenidos en el balance general \\
\hline
\end{tabular}

Fuente: Elaboración propia. 
Modelo de referencia operacional aplicado a una empresa de servicios... Herrera Vidal, German y Herrera Vega, Juan Carlos

\section{Cuadro 3}

\section{Problemas analizados en cadenas de suministros bajo el modelo SCOR}

\begin{tabular}{cc}
\hline Problemas & Autores \\
\hline Gestión de inventarios & Patiño (2008); López y Uricoechea (2011); \\
Olarte (2011); Pacheco y Rojas (2011). \\
Información concerniente a la demanda & Patiño (2008); Fontalvo et al. (2010); López y \\
Configuración de la cadena & Salazar y López (2009) \\
Cumplimiento de las ordenes & Fontalvo et al. (2010); Campos et al. (2012) \\
Costos logísticos & Fontalvo et al. (2010); Pacheco y Rojas 2011) \\
Rentabilidad & Fontalvo et al. (2010) \\
Logística reversa & Olarte (2011) \\
Capacidad & Salazar et al. (2012) \\
Calidad del producto final & Cortes y Ardila (2011) \\
Planeación de compras, despachos y operaciones & Ramírez y Rozo (2012)
\end{tabular}

Fuente: Elaboración propia.

\section{Cuadro 4 \\ Tipo de sector de aplicación del modelo SCOR}

\begin{tabular}{|c|c|}
\hline Sector & Autores \\
\hline Automotriz & $\begin{array}{l}\text { Patiño (2008); López et al. } \\
\text { (2011) }\end{array}$ \\
\hline Farmacéutico & Fontalvo et al. (2010) \\
\hline Textil & $\begin{array}{l}\text { Olarte (2011); Cortes y } \\
\text { Ardila (2011) }\end{array}$ \\
\hline Agrícola & Ramírez y Rozo (2012) \\
\hline Construcción & Campos et al. (2012) \\
\hline Energético & Salazar et al. (2012) \\
\hline Forestal & Salazar y López (2009) \\
\hline Comercial & Pacheco y Rojas (2011) \\
\hline
\end{tabular}

Fuente: Elaboración propia.

que en los últimos meses han aumentado en cantidad, pues cada día, el tema de mantenimiento preventivo en sitio toma más auge, representa un $10 \%$ de la producción total. (v) Servicio de suministros, este servicio comprende el suministro de repuestos para equipos eléctricos, tales como ventiladores plásticos, ventiladores de metal, impulsores, rotores, entre otros; y (vi) El suministro de equipos nuevos de las marcas de las cuales la compañía es taller autorizado, Siemens y Weg, lo que permite ofrecerle al cliente la dos opciones, una la de la reparación del equipo o dos el suministro de un equipo nuevo completamente garantizado por las marcas, este representa un $5 \%$ de los servicios prestados.

Los actores importantes de la cadena son los clientes locales y los regionales que comprenden otras ciudades de la costa, ambos se destacan por la cantidad de trabajos que envían y la frecuencia con la que lo hacen, la compañía, dispone actualmente de un portafolio amplio de clientes finales, integrado por empresas ubicadas en los más variados secto- 
res de la economía del país, este portafolio se identifica por proveer a empresas con un amplio reconocimiento en el mercado nacional y por un alto nivel de calidad en cuanto a los productos y servicios que ofrecen.

Otros actores importantes de la cadena de suministros son los proveedores, clasificados como locales y nacionales, los locales se encargan de suministrar y distribuir toda la gama de retenedores, rodamientos, tornillos, piezas de metal, arandelas, tuercas, accesorios metálicos, varillas roscadas, pinturas de esmalte, entre otros. Los proveedores nacionales suministran cables y alambres eléctricos de cobre y aleaciones de aluminio, aislantes eléctricos (fibras, barniz, esmalte, espagueti tubular) y conductores eléctricos.

La empresa realiza la recepción de los servicios por medio de los diferentes medios de comunicación que maneja con sus clientes, vía telefónica, vía e-mail, una vez recibido el servicio se procede al inicio de todas las labores propias para la entrega final del mismo, en taller, conforme al servicio a prestar, se solicitan los diferentes tipos insumos al área de gestión logística, quien es responsable de proveerle a producción todo los necesario en cuanto a materia primas para la realización de actividades del procesos productivo, al finalizar este proceso, es decir, cuando los insumos se encuentran en almacén se inician las diferentes actividades del proceso productivo, hasta llegar a la liberación de equipos, considerado como el procedimiento en el cual se libera el equipo del área de producción, dejándose listo para su entrega, para esta última actividad, se coordina con el cliente la forma de entrega ya sea enviándolo usando el sistema de transportadores o informando la disponibilidad para su retiro.

Considerando que en esta cadena de suministros se presentan los componentes y procesos claves del modelo SCOR, como es el caso de las interacciones y los diferentes procesos que intervienen desde la planificación, suministros, producción distribución y devolución. La empresa seleccionada servirá de insumo para el análisis y la evaluación de dicho modelo,

\subsection{Estado actual de la cadena de suministros}

La cadena de suministros está conformada por los proveedores, la empresa y los clientes, esta inicia aguas arribas desde la recepción del requerimiento de los clientes del determinado bienes y servicios, petición que es realizada por medio magnético, por teléfono, o por medio de contacto con el área comercial la cual transmita la información a producción para que este coordine la recepción del servicio, pasando por las compras realizadas a los proveedores locales o nacionales para la prestación del servicio, hasta finalmente realizar la entrega final del mismo, los cuales son transportados mediante vehículos propios de la empresa. En el Diagrama 2 se puede evidenciar el alcance de la cadena de suministros de la empresa caso estudio.

Para obtener un correcto diagnóstico de la situación actual presentada en la empresa caso de estudio, es preciso iniciar con el análisis de datos proporcionado en el desarrollo de la herramienta del análisis DOFA, en donde se ubicaron los factores concernientes a cada paráme- 


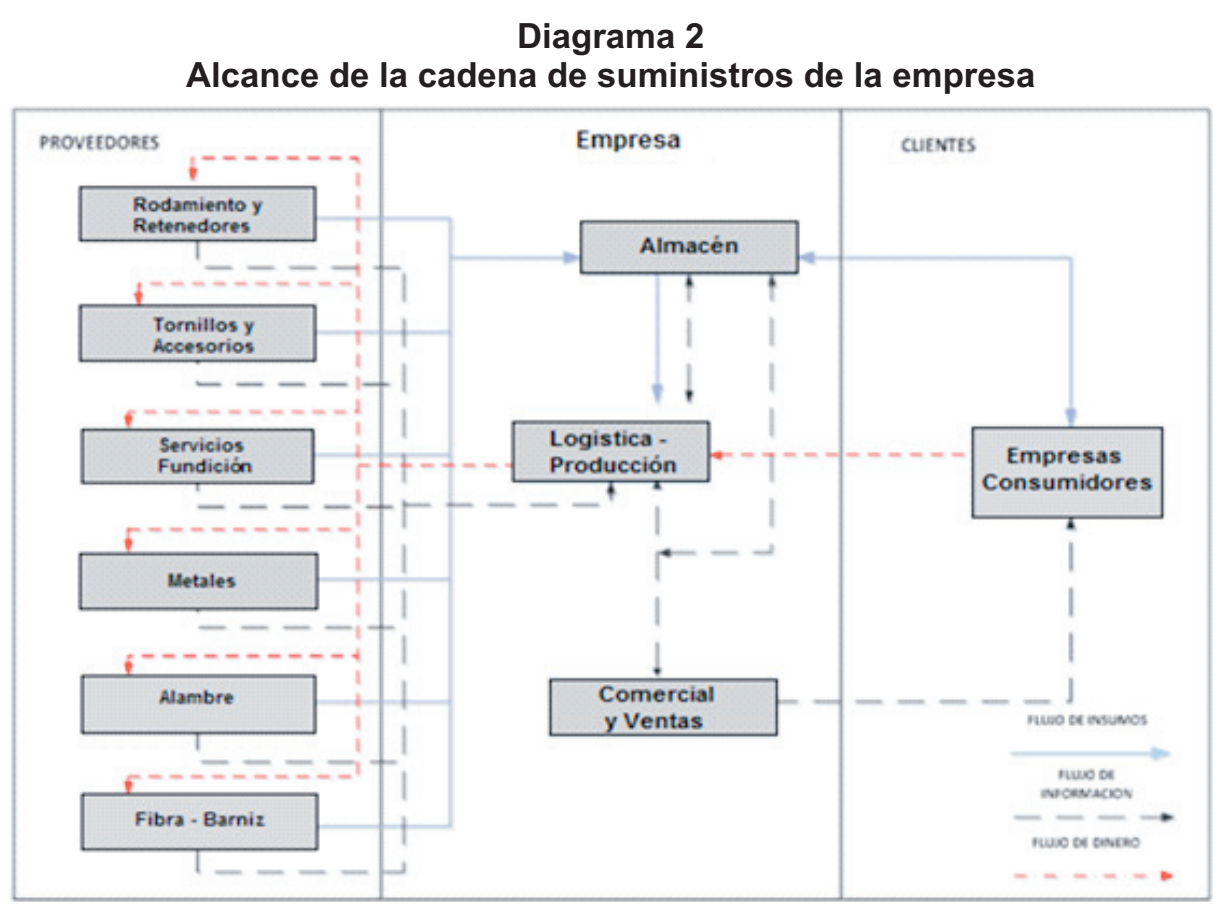

Fuente: Elaboración propia.

tro, como se evidencia en la matriz (Cuadro 5).

Teniendo en cuenta los aspectos internos como las debilidades, fortalezas, y los externos como las oportunidades y amenazas, es evidente la ineficacia de la cadena de suministros, la falta de planeación de la producción y la competencia desleal al igual que la llegada al país de equipos cada vez más accesible al público, la pérdida de clientes por no ofrecer oportunamente la reparación de equipo a falta de repuestos, preocupan seriamente a los directivos de la compañía; incluso tales debilidades y amenazas pueden afectar significativamente al reconocimiento local y nacional trayendo como consecuencia la disolución del apoyo otorgado por las marcas reconocidas de los equipos.

\section{Análisis del Modelo SCOR}

Teniendo como fundamento la parte metodológica del modelo SCOR, en la empresa caso de estudio dentro de su cadena de suministros se desarrolla un análisis de acuerdo a los diferentes niveles que lo componen.

\subsection{Análisis del nivel superior}

Dentro de los procesos que se encuentra en la cadena están los de planificación, abastecimiento, producción, distribución y retornos. Esta está definida 


\section{Cuadro 5 \\ Matriz DOFA de la empresa caso estudio}

Debilidades

- Ineficacia de la cadena de suministro

- Falta de metodologías para la planeación de la producción

- Falta un sistema de costeo para la producción

- Falta de proveedores locales de repuestos de calidad.

- Plataforma informática antigua

- Poca coordinación entre los departamentos internos de la compañía

- Inexistencia de mecanismos de control de procesos
Fortalezas

- Reconocimiento en el mercado local y nacional como empresa prestadora de servicios eléctricos industriales.

- Utilización de equipos y maquinaria avanzada tecnología en comparación con la que emplea la competencia

- Las competencias, habilidades y experiencia del personal que la labora en la compañía y por la cual esta se destaca.

Oportunidades

Amenazas

- La ventaja de las empresas industriales del

- La iniciación del tratado de libre comercio sector de enviar sus equipos a un taller certificado por marcas reconocidas.

- La ampliación de la refinería de Cartagena (ECOPETROL), en la ciudad y la puesta en marcha de esta sede de la industria petrolera.

- Las asesorías y capacitaciones ofrecidas por los proveedores y entes reconocidos de insumos al personal de la compañía.

- Aumento en los niveles tecnológicos en la industria Colombiana.

(TLC) en Colombia, trae al mercado motores y demás equipos eléctricos de calidad a un menor precio y en menor tiempo de entrega.

- La competencia desleal que cada vez baja de valor sus listas de precios para abarcar más el mercado.

- La competencia maneja un mayor stock (inventario) de repuestos, cuya disponibilidad disminuye tiempos de entrega y mejora eficacia del sistema.

- Competencia con mejores tiempos de entrega

- Las licitaciones de contratos con empresas reconocidas de diversos sectores de la economía del país, exigen la certificación en sistema de gestión ambiental y sistema de gestión de seguridad y salud ocupacional.

Fuente: Elaboración propia.

desde los proveedores nacionales hasta las empresas consumidoras a las cuales se les presta el servicio de reparación de equipos eléctricos de manera directa (Diagrama 3).

El modelo SCOR propone una serie de indicadores de gestión denominados KPIs (Key performance indicators), los cuales proveen a los procesos, medi- das de desempeño en los diferentes niveles contemplados en el mismo. Estos permitirán evidenciar de forma práctica, las mejoras que se ofrecerán en la cadena de suministros.

Los indicadores de primer nivel no se relacionan necesariamente con todos los procesos (Plan, aprovisionamiento, manufactura, distribución, devolución). 


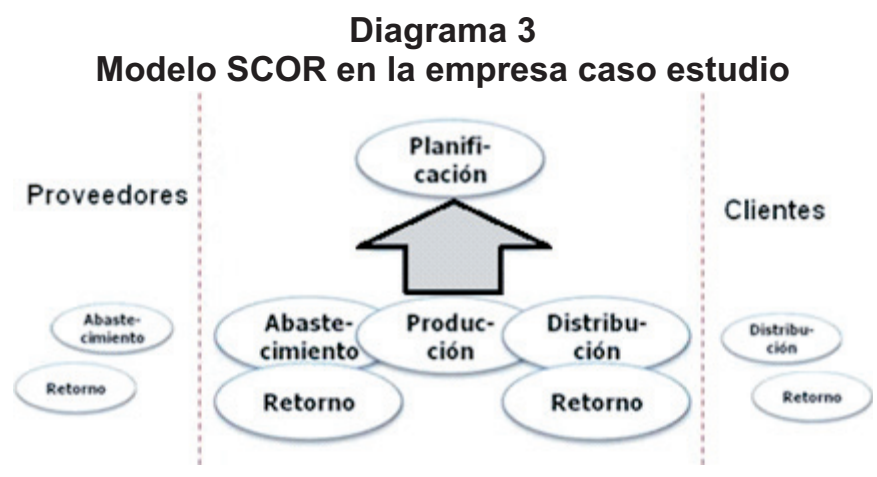

Fuente: Elaboración propia.

Los tres primeros (fiabilidad en el cumplimiento, flexibilidad y velocidad de atención) son puntos de vista externos, mientras que costo y activo son puntos de vista internos (Calderón y Lario, 2005:3).

Para la medición de estos indicadores, hay que tener en cuenta que, según la Supply Chain Council (2010), (i) La fiabilidad, mide el desempeño de la cadena de suministros en el proceso de entrega de repuestos, articulo o producto correcto, en condiciones aptas, con la calidad requerida, con la documentación e información pertinente. (ii) La velocidad de atención, se refiere a la velocidad con la cual la cadena de suministros provee productos a clientes tanto internos como externos, para ello, miden el tiempo empleado de una tarea particular dentro de la cadena de suministros desde que se inicia la actividad hasta que finaliza. (iii) La flexibilidad, hace referencia a la agilidad de la cadena de suministros ante cambio en el mercado, con el propósito de mantener o ganar ventajas competitivas. (iv) Los costos, hace referencia a los costos asociados a la operación y gestión de la cadena de suministros. (v) la gestión de activos, miden la efectividad financiera de la cadena de suministros, el manejo que se le da a los activos fijos o al capital de trabajo (Cuadro 6).

A partir de esto se expone la definición y la medición de tales atributos, aplicados en la gestión logística actual de la empresa caso de estudio (Cuadro 7). Los resultados obtenidos evidencian que existen condiciones en los rendimientos actuales encaminados a poder mejorar la empresa, desde el punto de vista externo e interno, viéndose reflejada en la gestión de los clientes, los costos y activos.

El análisis de este primer nivel permitió concluir que la administración de la cadena de suministro de la empresa mantenía un comportamiento bueno, pero existían factores susceptibles a mejoras tales como el aumento en el cumplimiento de las órdenes, incremento en la velocidad de entrega de los productos a los clientes, mayor flexibilidad para adaptarse con más rapidez a los cambios presentados en la demanda y se requiere de igual forma una mayor gestión sobre los activos, pero sobretodo de los costos operacionales, con el fin de aumentar las ganancias netas y por ende la rentabilidad de la empresa. 


\section{Cuadro 6 \\ Indicadores de primer nivel en la empresa}

\begin{tabular}{|c|c|c|c|c|c|}
\hline \multirow[t]{2}{*}{ Atributos de cambio } & \multicolumn{3}{|c|}{ Puntos de vista externos } & \multicolumn{2}{|c|}{ Puntos de vista internos } \\
\hline & Respuesta & Velocidad & Flexibilidad & $\begin{array}{l}\text { Costos de } \\
\text { gestión }\end{array}$ & $\begin{array}{l}\text { Gestión de } \\
\text { activos }\end{array}$ \\
\hline $\begin{array}{l}\text { Cumplimiento de entrega de } \\
\text { servicios }\end{array}$ & $x$ & & & & \\
\hline $\begin{array}{l}\text { Ciclo de tiempo de } \\
\text { cumplimiento de una entrega } \\
\text { de servicios }\end{array}$ & & $x$ & & & \\
\hline $\begin{array}{l}\text { Flexibilidad en la cadena de } \\
\text { suministros hacia arriba }\end{array}$ & & & $\mathrm{x}$ & & \\
\hline $\begin{array}{l}\text { Costo total de gestión de la } \\
\text { cadena de suministros }\end{array}$ & & & & $x$ & \\
\hline $\begin{array}{l}\text { Retorno de los activos fijos de } \\
\text { la cadena de suministros }\end{array}$ & & & & & $x$ \\
\hline
\end{tabular}

Fuente: Adaptado a partir de Supply Chain Council.

\section{Cuadro 7}

\section{Calculo de indicadores de gestión del primer nivel}

\begin{tabular}{clc}
\hline Indicador & \multicolumn{1}{c}{ Calculo del indicador } & $\begin{array}{c}\text { Rendimiento } \\
\text { Actual }\end{array}$ \\
\hline $\begin{array}{c}\text { Respuesta de la } \\
\text { cadena de suministros } \\
\text { Velocidad de atención }\end{array}$ & $\begin{array}{l}\text { Total de servicios entregados en cumplimiento perfecto/total } \\
\text { Suma de tiempo de ciclo actual de los servicios entrega- } \\
\text { dos/total de servicios entregados }\end{array}$ & $71,4 \%$ \\
Flexibilidad & $\begin{array}{l}\text { Número de días requeridos para alcanzar un 20\% de incre- } \\
\text { mento no planeados en los servicios }\end{array}$ & $10,0 \%$ \\
Costos & $\begin{array}{l}\text { Suma de costos por planear, abastecer, entregary devolver } \\
\text { Ingresos de la cadena - costo de ventas - costo de ges- }\end{array}$ & $77,0 \%$ \\
Gestión de activos & $37,0 \%$ \\
\hline
\end{tabular}

Fuente: Elaboración propia.

\subsection{Análisis nivel de configuración}

Este segundo nivel, permite describir de forma más amplia cada proceso de acuerdo a su categoría, para efectuar la configuración de la cadena de suministros, es necesario contrastar los procesos propuestos por el modelo SCOR, contra los que se realizan en la empresa, para poder aplicar correctamente las métricas y mejores prácticas propuestas.

La empresa presenta un sistema de producción basado en la política fabricación bajo pedido, por lo tanto todos los 
Modelo de referencia operacional aplicado a una empresa de servicios... Herrera Vidal, German y Herrera Vega, Juan Carlos

procesos pertenecientes a la cadena de suministros, están alineados de esta manera, abastecimiento bajo pedido y distribución bajo pedido, la devolución se adapta a la definición de la sub-categoría de producto para mantenimiento general y reparación.

A partir de esto de las 26 categorías de procesos que plantea el modelo SCOR, se hace la configuración de la cadena de la empresa, al cual corresponden 5 a Plan, 1 a Aprovisionamiento, 1 a Manufactura, 1 a Distribución y 2 a Devolución (1 de Aprovisionamiento y 1 de Distribución) (Diagrama 4.).

Con base a esta configuración y a lo que actualmente sucede en la empresa, se puede inferir que la planeación es un proceso en el cual la empresa no tiene plenamente identificados ni desarrollados los parámetros necesarios para lograr una ventaja real y competitiva, por lo que se evidencia la falta de planeación en procesos como compras y producción, dado que no se han determinado, los mecanismos requeridos para realizar este proceso de forma completa dentro de la cadena de suministro.

Geográficamente se pueden establecer dos procesos importantes que son el abastecimiento (suministro) y la distribución o entrega, en el cual no se han identificado oportunidades de mejora puesto que: el abastecimiento de las materias primas de proveedores internacionales tales como el proveedor de alambre magneto y de material aislante; identificados por el modelo SCOR como P4, S1, M1, D4, DR2, SR1; llega a las sucursales en Colombia mediante un proceso de importación cuyos procedimientos legales son inevitables y son llevados a cabo única y exclusivamente por estos entes. El resto de proveedores nacionales, proveedor de productos químicos, proveedor repuestos (balineras, retenedores, sellos mecánicos) llevan a cabo los siguientes procesos S1, M1, D4, DR1,

\section{Diagrama 4 \\ Configuración de las categorías de los procesos de la cadena de la empresa}

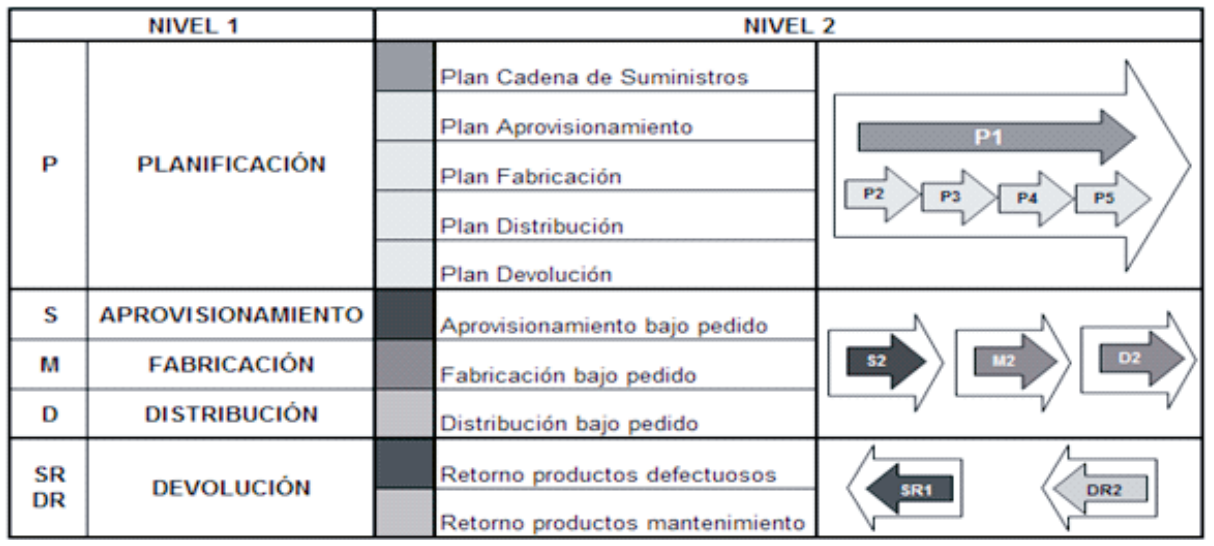

Fuente: Elaboración propia. 
DR3, en los cuales la empresa es parte fundamental, sobre todo en actividades de devolución y entrega de pedidos. Al igual que los proveedores internacionales han sido seleccionados por la calidad ofrecida por sus productos (Diagrama 5).

La planeación de la empresa está determinada por los elementos establecidos por el modelo SCOR P1, P2, P3, P4, $\mathrm{P} 5$, donde se determinan todos y cada uno de los requerimientos de la cadena de suministro al igual que su gestión. La producción identificada por el modelo SCOR como M2, es realizada tal y como ha sido descrita en las generalidades del caso de estudio.

La distribución de servicios desde empresa, hasta los respectivos clientes, D2, se realiza a través de una flota propia de vehículos y personal vinculado activa- mente a esta, debido a que se cuenta con la infraestructura necesaria para cumplir con las entregas de servicios solicitados, se descarta cualquier iniciativa de mejora en este aspecto.

Los indicadores (performance metrics) del segundo nivel, de igual forma contemplan los atributos del modelo SCOR, como son: fiabilidad en el cumplimiento, capacidad de respuesta, agilidad, costos, y manejo de activos. Permitiendo realizar un diagnóstico a las métricas del primer nivel, y ayudando a identificar errores o vacíos presentados con anterioridad. En el Cuadro 8 se evidencia la medición de los indicadores en la cadena de suministros de la empresa, teniendo en cuenta atributos de rendimiento como:

(i) Perfecto cumplimiento del servicio, (ii) Ciclo de tiempo de cumplimiento del

\section{Diagrama 5 \\ Diagrama geográfico de la cadena de suministros}

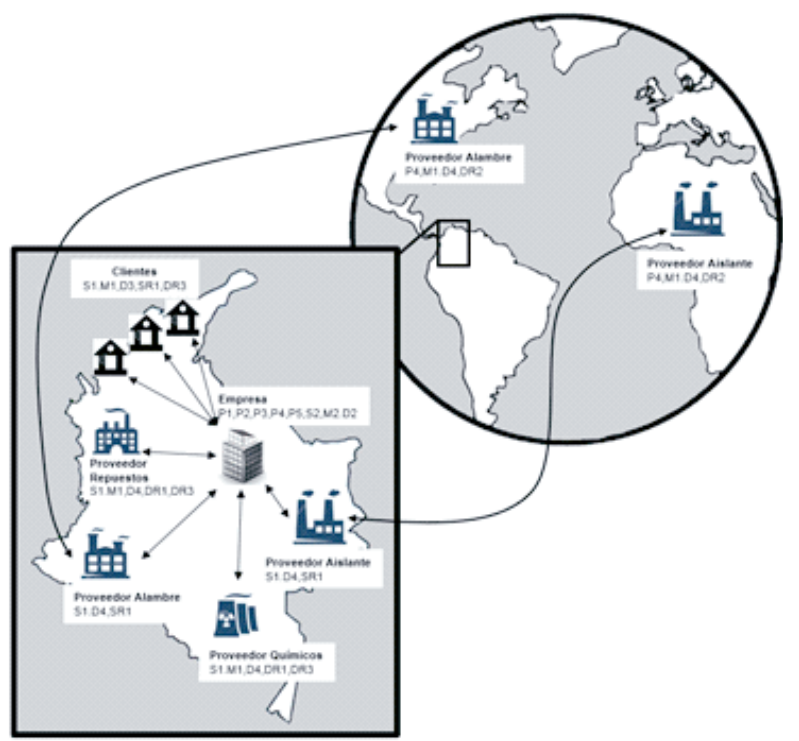

Fuente: Elaboración propia. 
Modelo de referencia operacional aplicado a una empresa de servicios... Herrera Vidal, German y Herrera Vega, Juan Carlos

servicio, (iii) Flexibilidad, (iv) Costo de gestionar la cadena de suministros y (v) Retorno de capital de trabajo. Dado que los atributos no pueden ser medidos, las métricas son usadas para calcular el rendimiento que cada uno de estos, y así definir una estrategia para su óptima operación. Esos se calculan teniendo en cuenta los cálculos métricos del Cuadro 8.

\subsection{Análisis nivel de elementos}

En el tercer nivel se representan los distintos procesos de la cadena de suministros de manera más detallada descomponiendo las categorías en elementos de procesos (Process elements). Estos se presentan en secuencia lógica (con rectángulos y flechas) con entradas (inputs) y salidas (outputs) de información y materiales. Además, en de esto se evalúa el rendimiento de cada proceso y elemento mediante índices (Process performance metrics), de manera que se encuentren las diferencias de rendimiento entre los procesos y elementos de la cadena de suministros. (Calderón y Lario, 2005:6).

Este nivel permite visualizar de forma clara y completa las características con las que cuenta cada uno de los procesos contenidos en la cadena de suministro, estableciendo las mejores prácticas aplicables y las métricas de rendimiento respectivas lo que admitirá una visión generalizada de la cadena de suministro.

Teniendo en cuenta el proceso de planificación, existen diferentes tipos de planes dependiendo de los agentes que conforma la cadena, dado lo anterior para el tercer nivel se encuentra el plan general de la cadena de suministros (P1), conformado por los siguientes elementos 0

\section{Cuadro 8 \\ Calculo de indicadores de gestión del segundo nivel}

\begin{tabular}{llc}
\hline \multicolumn{1}{c}{ Atributo de Rendimiento } & \multicolumn{1}{c}{ Métricas } & Valor Métrica \\
\hline \multirow{2}{*}{$\begin{array}{llc}\text { Perfecto cumplimiento del } \\
\text { servicio }\end{array}$} & Porcentaje de servicios en su totalidad & $95,23 \%$ \\
& Rendimiento de entrega al cliente & $72,82 \%$ \\
& Perfecto estado & $100 \%$ \\
\hline $\begin{array}{l}\text { Ciclo de tiempo de cumplimiento } \\
\text { del servicio }\end{array}$ & Ciclo de tiempo del suministro & 72 horas \\
\hline Flexibilidad & Ciclo de tiempo de entrega & 120 horas \\
\hline & Flexibilidad de las compra hacia arriba & 7 días \\
Costo de gestionar la cadena de & Costo de planificar & USD 747 \\
suministros & Costo de distribuir & USD 16.985 \\
& Costo de devolver & USD 3.397 \\
Retorno de capital de trabajo & Cuentas por pagar & USD 51 \\
\cline { 2 - 3 } & Inventario & USD 20.382 \\
\hline
\end{tabular}

Fuente: Elaboración propia. 
actividades de procesos: (i) Identificar, priorizar y consolidar los requerimientos de la cadena de suministros - P1.1, (ii) Identificar, evaluar y consolidar los recursos de la cadena de suministros $\mathrm{P} 1.2$, (iii) Balancear los recursos con los requerimientos de la cadena de suministros - P1.3, (iv) Establecer y comunicar el plan de la cadena de suministros - P1.4. y el plan de aprovisionamiento (P2), conformado por: (i) Identificar, priorizar y consolidar los requerimientos del producto P2.1, (ii) Identificar, evaluar y consolidar los recursos disponibles - P2.2, (iii) Balancear la asignación de recursos a los requerimientos de los productos - P2.3, (iv) Establecer y comunicar el plan de abastecimiento - P2.4.

En cuanto al proceso de Aprovisionamiento, se encuentran el aprovisionamiento bajo pedido (S2), conformado por cinco elementos o actividades de procesos como son: (i) Fechas de entrega de productos - S2.1, (ii) Recibimiento del producto - S2.2, (iii) Verificación del producto - S2.3, (iv) Transferencia del producto - S2.4 y (v) Autorización de pago a proveedores - S2.5.

El proceso de Fabricación se da bajo pedido (M2), y está conformada por seis elementos o actividades de procesos como son: (i) programación de actividades de producción - M2.1, (ii) Requerimientos de la producción - M2.2, (iii) Ejecución de la producción - M2.3, (iv) Empaque del producto terminado - M2.4, (v) Almacenamiento del producto terminado - M2.5 y (vi) Preparación de la documentación sobre la producción - M2.6.

En cuanto al proceso de Distribución, la distribución bajo pedido (D2), agrupan siete elementos claves de pro- cesos como son: (i) Solicitud cotización del servicio - D2.1, (ii) Recibir producto en la fábrica - D2.8, (iii) Recoger producto D2.9, (iv) Generar documentación para el transporte - D2.11, (v) Enviar producto D2.12, (vi) Recibir y verificar el producto por parte del cliente - D2.13 y (vii) Facturar - D2.15.

En la Devolución, se encuentran diferentes tipos de retornos dependiendo de los agentes que intervienen, si es de la fábrica hacia el proveedor (SR) o si de parte del cliente hacia la fábrica (DR), dentro de cada uno se presenta una logística inversa ya sea por productos en caso de defecto, reparación o productos en exceso. Dado lo anterior para el tercer nivel se presenta una devolución denominada, retorno de productos al proveedor (SR2), conformada por: (i) Identificar la condición de producto - SR2.1 y (ii) Autorización de retorno de producto - SR2.3. Dentro del proceso de devolución de los clientes hacia la fábrica, se presenta de igual forma el retorno de productos por parte del cliente (DR2), compuesta por: Autorizar retorno de producto - DR2.1 y (ii) Recibir producto - DR2.3 (Diagrama 6).

En síntesis, dentro del análisis hecho se puede detallar que existen actividades planteadas por el Modelo SCOR que dentro de la empresa no se están ejecutando, evidenciando así deficiencias en la gestión de la cadena de suministro en aspectos como la planeación y el control de los procesos.

Luego de la identificación de las fallas encontradas en la empresa, se propusieron las acciones tendientes a mejorar esas falencias en la cadena de suministros de la empresa: 


\section{Diagrama 6 \\ Elementos del tercer nivel de la cadena de suministros}

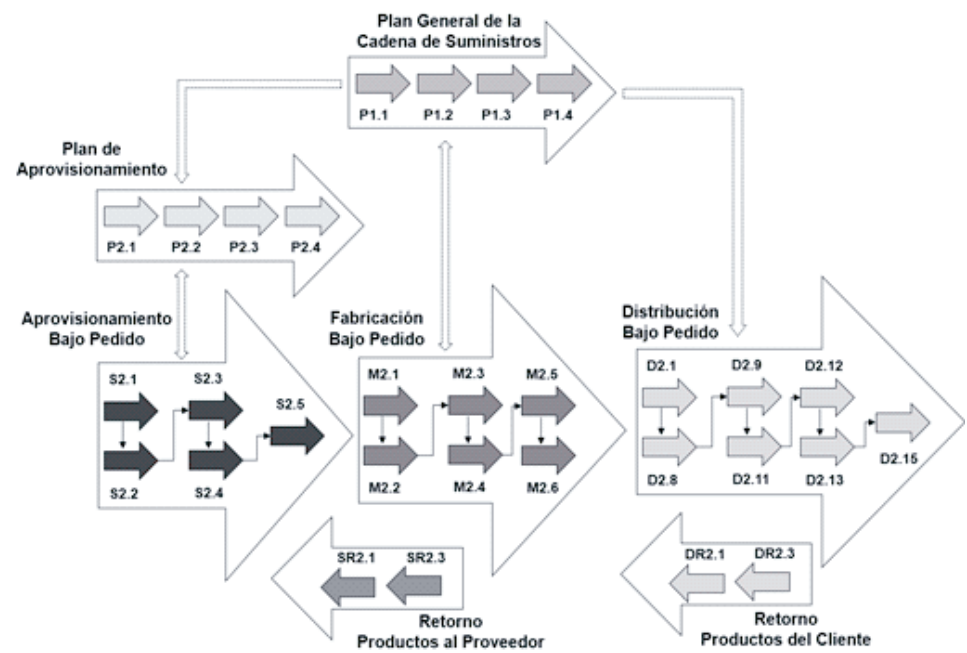

Fuente: Elaboración propia.

1. El desarrollo de un sistema avanzado para la planeación de la cadena de suministros, que permitan y faciliten la visibilidad de los requerimientos de cada uno de las partes o eslabones que la conforman.

2. Revisar la rentabilidad de alguno de los servicios ofrecidos a los clientes.

3. Utilizar indicadores clave de desempeño (KPl's) para evaluar y controlar el desempeño de los procesos.

4. Fortalecer el intercambio de información en tiempo real entre los miembros de la cadena de suministro.

5. Reducir el costo del inventario en stock sin sacrificar la capacidad de respuesta y evitar contar con inventarios altos de productos que no tienen suficiente rotación, también plantear la posibilidad de aplicar acuerdos con los proveedores para gestionar el compra en volúmenes que permitan cubrir las necesidades de periodos de tiempo establecidos más allá de comprar día a día.

6. Implementar mecanismos que puedan medir y evaluar efectivamente la gestión de los proveedores y el servicio al cliente así como establecer una lista de criterios para seleccionar. $Y$ (vii) Identificar, proponer y evaluar los tiempos de entrega de los proveedores de los insumos que le son solicitados incluyendo la calidad de los mismos de tal forma que esta información sirva como base principal para pactar el tiempo de cumplimiento y finalización de los trabajos de reparación y rebobinado de motores.

\section{Conclusiones}

De los resultados encontrados en el análisis de los casos investigados, en 
cuanto a la aplicación del modelo SCOR, se reflejan la poca aplicabilidad de este en el sector eléctrico industrial, viéndose evidenciados problemas relacionados con el cumplimiento de las órdenes y la calidad del producto final. Aparte de esto en el análisis logrado mediante la matriz DOFA, se hace evidente la ineficacia de la cadena de suministros y la falta de planeación de la producción. Es por esto que el presente artículo logra mostrar la importancia que hoy en día tiene el adoptar e implementar una metodología que abarque toda la complejidad de administrar eficientemente la cadena de suministro de una organización, y que a su vez permita poder identificar los falencias que se presentan en cada uno de sus procesos, mediante la aplicación de un modelo referencial, que pueda dar respuestas oportunas cada uno de los problemas encontrados.

El modelo SCOR, permitió la identificación y definición del alcance de la cadena de suministro de la empresa, donde se visualizaron los procesos que participan, el flujo de información e insumos necesarios para el desarrollo de las actividades en la prestación de servicio. Además, facilitó controlar de forma más eficiente los procesos de la cadena de suministro, mejorando el desempeño de los mismos, logrando el seguimiento y reconfiguración de las actividades operativas por medio de la identificación del rendimiento, valorando cada atributo por medio de las métricas establecidas; logrando diseñar un plan de acción de tal manera que se pueda aumentar la utilidad y elevar la satisfacción del cliente.

Para futuras investigaciones seria provechoso aplicar el modelo en cadenas de suministros con varios niveles de aprovisionamiento y varios de distribución, además medir la cadena teniendo en cuenta los otros atributos de cambio que propone el modelo SCOR.

\section{Referencias bibliográficas}

Bolstorff, Peter y Rosenbaum, Robert (2012), Supply chain excellence: A handbook for dramatic improvement using the SCOR model. Tercera Edición. New York. Amacom - American Management Association. ISBN 08144-1771-X.

Burns, J.F. y Sivazlian, B.D. (1978), Dynamic analysis of multi-echelon supply systems. Computers \& Industrial Engineering. Department of Industrial and Systems Engineering. University of Florida. USA. Volumen 2, Issue 4, pp. 181-193.

Calderón José y Lario Francisco (2005), Análisis del modelo SCOR para la gestión de la cadena de suministro. IX Congreso de Ingeniería de Organización. Disponible: http://adingor.es/ congresos/web/uploads/cio/cio2005/ cadena_suministros//41.pdf. (Fecha de consulta: 07/06/2015).

Campos, José; Cruz, Claudia y Sánchez, Juan (2012). Diagnóstico basado en el Modelo SCOR para la cadena de suministro de la empresa Matecsa S.A. Revista Avances Investigación en Ingeniería. Universidad Libre. Volumen 9, Issue 12, pp. 94-100.

Cortes, Luisa y Ardila Pedro (2011), Diagnóstico a las pymes productoras de cuero bajo el modelo SCOR con el fin de determinar el estado actual. Universidad de La Salle. Disponible: http://repository.lasalle.edu.co/handle/10185/2928. (Fecha de consulta: 14/06/2015). 
Modelo de referencia operacional aplicado a una empresa de servicios... Herrera Vidal, German y Herrera Vega, Juan Carlos

Chandra, Charu y Kumar, Sameer (2000), Supply chain management in theory and practice: a passing fad or a fundamental change. Industrial Management \& Data Systems, Volumen 100 , Issue 3, pp. $100-114$.

Fontalvo, Tomás; De la Hoz, Efraín y Cardona, Rojas (2010), Diseño de un plan de mejoramiento para la cadena de suministro de la empresa Drolitoral S.A. Aplicando el modelo SCOR. Revista soluciones de posgrados EIA. Escuela de Ingeniería de Antioquia. Volumen 3, Issue 6, pp. 33-54.

Forrester, Jay (1961), Industrial dynamics. Massachusetts Institute of Technology. Primera Edición. Editorial Wiley. New York.

Herrera, German (2014), Análisis de Modelos de Planificación Colaborativa en la Cadena de Suministros: Una Revisión de la Literatura. Latin American and Caribbean Conference for Engineering and Technology. LACCEI. Ecuador. Disponible en: http://www. laccei.org/LACCEI2014-Guayaquil/ RefereedPapers/RP022.pdf. (Fecha de consulta: 10/07/2015).

Huan, Samuel; Sheoran, Sunil y Wang, Ge. (2004), A review and analysis of supply chain operations reference (SCOR) model. Supply chain management: An International Journal. Volumen 9, Issue 1, pp. 23-29.

Lambert, Douglas; Cooper, Martha y Pagh, Janus (1998), Supply chain management: Implementation issues and research opportunities. The international journal of logistics management. Volumen 9, Issue 2, pp. 1-20.

Lario Francisco y Pérez David (2005), Gestión de Redes de Suministro (GRdS): sus Tipologías y Clasificaciones. Modelos de Referencia Conceptuales y Analíticos". IX Congreso de ingeniería de organización. Gijón, 8 y 9 de septiembre de 2 .
Lee Hau y Billington Corey. (1993), Material management in decentralized supply chains. Operations research. Volumen 41, Issue 5, pp. 835-847.

López, Sebastián y María, Uricoechea (2011), Propuesta para la optimización del rendimiento de la cadena de suministro para la empresa Autocom S.A. a partir de la utilización del modelo SCOR y la integración de procesos mediante tecnologías de información. Pontificia Universidad Javeriana. Disponible:http://hdl.handle. net/10554/7446. (Fecha de consulta: 13/06/2015).

Min, Soonhong y Mentzer, John. (2000), The role of marketing in supply chain management. International Journal of physical distribution \& logistics management. Volumen 30, Issue 9, pp. 765-787.

Olarte, Michelle (2011), Propuesta de diseño de un modelo de logística reversa para el sector textil colombiano bajo la metodología SCOR. Pontificia Universidad Javeriana. Disponible: http://hdl.handle.net/10554/7412. (Fecha de consulta: 13/06/2015).

Pacheco, Amparo y Rojas, Manuel (2011), Análisis del modelo SCOR para aplicación en el proceso de empaque de granos en la cadena de suministros de los almacenes YEP. Pontificia Universidad Javeriana. Disponible: http://hdl.handle.net/10554/ 7402. (Fecha de consulta: 13/06/ 2015).

Patiño, Alejandro (2008), Análisis del Modelo SCOR y su aplicación a una cadena de suministro del sector del automóvil. Universidad Politécnica de Valencia (UPV). Disponible en: https:// riunet.upv.es/handle/10251/12380. (Accedido el 14/06/2015).

Ramírez, Leila y Rozo, Diego (2012), Diseño de la gestión logística para la cadena productiva de la papa criolla en el mu- 
nicipio de El Rosal Cundinamarca. Caso proveedores de insumos. Revista Gestión y Sociedad. Universidad de La Salle. Volumen 5, Issue 9, pp. 133-145.

Salazar, Hugo y López César (2009), Propuesta metodológica para la aplicación del modelo Supply Chain Operations Reference. Revista Ingeniería. Universidad Distrital Francisco José de Caldas. Volumen 14, Issue 2, pp. 34-41.

Salazar, Fernando; Cavazos, Judith y Martínez, José (2012), Metodología basada en el Modelo de Referencia para Cadenas de Suministro para Analizar el Proceso de producción de Biodiesel a partir de Higuerilla. Revista Información Tecnológica. Volumen 23, Issue 1, pp. 47-56.
Stevens, Graham (1989), Integrating the supply chain. International Journal of physical distribution \& materials management. Volumen 19, Issue 8 , pp.3-8.

Supply Chain Council (2004), Supply chain operations reference model (SCOR) 6.0. Disponible en: http:// www.supply-chain.org. (Fecha de consulta: 12/07/2015).

Supply Chain Council (2006), Supply chain operations reference model (SCOR) 8.0. Disponible en: http:// www.supply-chain.org. (Fecha de consulta: 09/07/2015).

Supply Chain Council (2010), Supply chain operations reference model (SCOR) 10.0. Disponible: http://www. supply-chain.org. (Fecha de consulta: 11/07/2015 
